\title{
Effects Of Methylmercury And Retinol Palmitate Co- Administration In Rats During Pregnancy And Breastfeeding: Metabolic And Redox Parameters In Dams And Their Offspring
}

Espitia Pérez, Pedro; Albino Suelen Marin, Suelen Marin; Da Rosa, Helen Tais; Silveira, Alexandre Kleber; Espitia Pérez, Lyda; Brango, Hugo; Moraes Pompéu, Diogo; Hermann Silva, Paolla Rissi; Mingori Rodrigues, Moara; Barreto, Fabiano; Kunzler, Alice; Gelain, D. P; Schnorr, Carlos Eduardo; Moreira Fonseca, José Cláudio

\section{Abstract}

Ubiquitous low-dose methylmercury ( $\mathrm{MeHg}$ ) exposure through an increased fish consumption represents a global public health problem, especially among pregnant women. A plethora of micronutrients presented in fish affects $\mathrm{MeHg}$ uptake/distribution, but limited data is available. Vitamin A (VitA), another fish micronutrient is used in nutritional supplementation, especially during pregnancy. However, there is no information about the health effects arising from their combined exposure. Therefore, the present study aimed to examine the effects of both $\mathrm{MeHg}$ and retinyl palmitate administered on pregnant and lactating rats in metabolic and redox parameters from dams and their offspring. Thirty Wistar female rats were orally supplemented with $\mathrm{MeHg}(0,5 \mathrm{mg} / \mathrm{kg} /$ day $)$ and retinyl palmitate $(7500 \mu \mathrm{g} R \mathrm{RA} / \mathrm{kg} /$ day) via gavage, either individually or in combination from the gestational day 0 to weaning. For dams (150 days old) and their offspring (31 days old), glycogen accumulation (hepatic and cardiac) and retinoid contents (plasma and liver) were analyzed. Hg deposition in liver tissue was quantified. Redox parameters (liver, kidney, and heart) were evaluated for both animals. Cytogenetic damage was analyzed with micronucleus test. Our results showed no general toxic or metabolic alterations in dams and their offspring by MeHg-VitA coadministration during pregnancy and lactation. However, increased lipoperoxidation in maternal liver and a disrupted pro-oxidant response in the heart of male pups was encountered, with apparently no particular effects in the antioxidant response in female offspring. GST activity in dam kidney was altered leading to possible redox disruption of this tissue with no alterations in offspring. Finally, the genomic damage was exacerbated in both male and female pups. In conclusion, low-dose MeHg exposure and retinyl palmitate supplementation during gestation and lactation produced a potentiated pro-oxidant effect, which was tissue-specific. Although this is a pre-clinical approach, we recommend precaution for pregnant women regarding food consumption, and we encourage more epidemiological studies to assess possible modulations effects of MeHg-VitA co- 
administration at safe or inadvertently used doses in humans, which may be related to specific pathologies in mothers and their children.

\section{Keywords}

Co-Exposure; Methylmercury; Oxidative Stress; Pregnancy; Vitamin A. 